
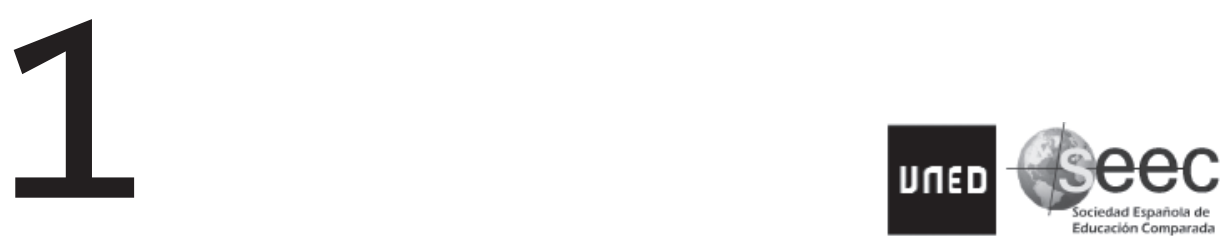

\title{
LA EDUCACIÓN INFANTIL EN \\ ALEMANIA, ESPAÑA, FRANCIA E \\ INGLATERRA. ESTUDIO COMPARADO
}

\section{Pre-primary education in Germany, Spain, France and England. Comparative Study}

Vicente Llorent

\section{RESUMEN}

La educación infantil no ha sido en absoluto ajena a la fuerte evolución que, durante las últimas décadas, han sufrido las sociedades europeas en general y las familias en particular. Esta etapa educativa ha pasado a realizar un importante papel de apoyo a las familias, yendo más allá de una mera función asistencial, pretende llevar a cabo una esencial misión educativa. Conscientes de la importancia de los primeros años de vida para el desarrollo integral del menor, las administraciones públicas de Alemania, España, Francia e Inglaterra han puesto especial interés en la atención a la infancia. Tras realizar un estudio comparado sobre la educación infantil implantada en estos países, comprobamos como sus políticas relativas a la educación infantil se basan en enfoques claramente diferenciados. Su

* Universidad de Sevilla. 
historia, contextos, valores, creencias y situación socioeconómica inciden fuertemente en las decisiones que cada uno de ellos ha adoptado.

PALABRAS CLAVE: Kindergarten, Educación infantil, École maternelle, Nursery, Playgroups.

\begin{abstract}
Early childhood education is not at all alien to the strong evolution over the past decades European societies have suffered in general and families in particular. This stage has come to perform an important role in supporting families, going beyond a mere function of care, intended to carry out an essential educational mission. Aware of the importance of early life for the integrated development of children, governments of Germany, Spain, France and England have a special interest in child care. After making a comparative study on child rearing practices implemented in these countries, we check that their policies on early childhood education are based on distinct approaches. Its history, contexts, values, beliefs and socioeconomic status strongly influence the decisions that each has adopted.
\end{abstract}

KEY WORDS: Kindergarten, Pre-primary education, École maternelle, Nursery, Playgroups.

$* * * * *$

\title{
INTRODUCCIÓN
}

Tanto el concepto y significado que cada sociedad tiene de la infancia como la valoración que hace de ella varían en función de la situación social, política y económica que mantenga. En este sentido, la educación infantil no ha sido en absoluto ajena a la fuerte evolución que, durante las últimas décadas, han sufrido las sociedades europeas en general y las familias en particular. El indudable influjo de los evidentes cambios operados en la mentalidad de los ciudadanos, en la legislación, en las condiciones laborales de los trabajadores (horarios, distancias entre hogar y lugar de trabajo, salarios...) se ha dejado notar. Del mismo modo, nos encontramos ante una serie de importantes transformaciones en la estructura familiar, en el desarrollo del estado del bienestar, en la incorporación de la mujer al mercado de trabajo y en las políticas igualitarias en cuanto al género. 
Todas estas alteraciones de aspectos antaño tradicionales, han propiciado una mayor participación de diversas instituciones educativas en pro de coadyuvar a la labor educativa que frecuentemente sobrepasa con creces la capacidad real educativa de muchas familias. Este proceso de transformación del medio familiar, laboral y social, está provocando un cada vez mayor protagonismo de instituciones que complementan la labor educativa de la familia, proporcionando al niño experiencias básicas que van a incidir decisivamente en su desarrollo y primeros aprendizajes.

Conscientes de la importancia de los primeros años de vida para el desarrollo integral del menor, las administraciones públicas de los países que vamos a estudiar han puesto especial interés en la atención a la infancia. Los amplios cuidados que requieren los más pequeños van más allá de la principal institución implicada, la familia, siendo fundamental el papel de los centros de educación infantil.

Una educación infantil y una atención a la infancia con un elevado nivel de calidad suponen grandes beneficios para sus destinatarios directos y para la sociedad, completando el papel central de la familia. Sientan las bases para la adquisición del lenguaje, el éxito del aprendizaje permanente, la integración social, el desarrollo personal y la capacidad de empleo. Aspectos sumamente beneficiosos para las fases ulteriores del aprendizaje, aumentando la equidad de los resultados educativos y reduciéndose los costes para la sociedad en pérdida de talento y gasto público en bienestar, sanidad e incluso justicia. Entre las conclusiones del Consejo sobre educación infantil y atención a la infancia destaca «ofrecer a todos los niños la mejor preparación para el mundo de mañana» (DIARIO OFICIAL DE LA UNIÓN EUROPEA, 15.6.2011: 175/8).

La educación infantil contribuye al desarrollo integral del alumno y coadyuva a una mejor adaptación del alumno a la vida colectiva. La gran demanda de los padres se ve respondida por una educación que, además, garantiza la atención y el cuidado de sus hijos: "La escuela infantil es, después de la familia, el agente educativo más idóneo por el alto grado de evolución alcanzado como institución educadora, y es también uno de los principales elementos de protección de la infancia» (SÁNCHEZ, 2008: 77).

La importancia de la educación infantil no sólo radica en los aspectos cognoscitivos sino que también es clave el desarrollo afectivo. La 
experiencia nos dice que hay claras diferencias entre los niños que están educados emocionalmente y los que no. Entre los factores que intervienen en el desarrollo de las respuestas emocionales se encuentran el temperamento, los padres y la educación, y es en este último factor donde la escuela puede tener un papel decisivo (TERCERO PUYOL y ROVIRA BALLABRIGA, 2008: 4).

Dada la diversidad de términos utilizados para la denominación de la etapa educativa objeto del presente estudio y las dificultades de su acotación semántica, conviene que procedamos sin más dilación a la delimitación de las dos expresiones más usuales en el ámbito de la Unión Europea (UE), los de «educación preescolar» y «educación infantil». El primero, más restrictivo, hace alusión a la educación que se imparte en determinadas edades con el objeto de preparar al alumno para su ingreso en el nivel de educación primaria. Mientras que el segundo, más amplio, comprende al primero y se conforma como una etapa educativa con entidad propia e independiente de la siguiente, en la que se incluyen niños con menos de un año de vida. Así pues, consideramos a la educación infantil como el cuidado, atención y educación que recibe el niño en el periodo anterior a la educación primaria (TORRES SÁNCHEZ y GONZÁLEZ FARACO, 2008: 50).

Generalmente, esta etapa educativa tiene un carácter no obligatorio. $\mathrm{Su}$ oferta se diversifica entre centros públicos y/o privados y escolares o no escolares, dependiendo de cada país en cuestión. Pero es tanta la diversidad de alumnado existente que la oferta educativa no se debe limitar a una oferta de plazas escolares si lo que se desea es ofrecer una verdadera igualdad de oportunidades para todos los niños (SÁNCHEZ, 2007).

Desde hace tiempo, y con demasiada frecuencia, se vienen calificando a los centros de educación infantil como meros «aparcamientos» de niños, refiriéndose al tipo de función que a éstos se les asigna y, de un modo indirecto, a la posible despreocupación de los padres por la formación que en ellos reciben sus hijos. En las últimas décadas, la sensibilidad social sobre el tema en cuestión ha sufrido un importante cambio en los países de la UE. Son cada vez más los partidarios de un modelo de escuela en el que se considere a la totalidad de este periodo como una etapa integrada en el resto del sistema educativo, cuyo fin no se centre en el cuidado y custodia los hijos, sino que por el contrario, se imparta una educación específica y diferenciada, a unos alumnos con características propias. En este sentido han evolucionado las políticas educativas de los países miembros de la UE. 
La educación infantil no constituye un espacio y un tiempo para la transmisión de enseñanzas y conocimientos, sino más bien un periodo donde apoyar, favorecer y potenciar el pleno desarrollo integral de los alumnos. Debería recibir la atención prioritaria de los gobiernos responsables, a través de leyes, políticas y facilitación de recursos. Una generosa inversión en estas primeras edades ayudaría a prevenir ulteriores problemas, en momentos en los que es más difícil intervenir y sin duda más costoso, tanto social como económicamente (ASOCIACIÓN DE MAESTROS ROSA SENSAT, 2004: 5).

A continuación procederemos a la comparación de distintos aspectos de la educación infantil que se está llevando a cabo en Alemania, España, Francia e Inglaterra, resaltando aquellas cuestiones que estimamos más interesantes.

\section{DESTINATARIOS Y CARÁCTER ${ }^{1}$}

La educación infantil en Alemania está muy poco extendida no tiene un carácter obligatorio. Apenas existen normativas que la regulen, ni planes de estudios para los centros. Las directrices que emanan de la Administración son muy generales y su cumplimiento no es de obligado cumplimiento. En los centros de educación infantil se pretende que los niños aprendan jugando voluntariamente y no por obligación, así se desarrollan su curiosidad y gusto por el aprendizaje, motivo por el que lo aprendido es más resistente al olvido.

La inmensa mayoría de los niños con edades comprendidas entre los 3 y los 6 años están inscritos en los denominados kindergarten. Estas instituciones carecen de un currículo formal establecido, se trata de una educación de carácter informal aunque con una clara orientación educativa. A pesar de que estos centros no son gratuitos existen largas listas de espera en algunas instituciones del oeste del país. En ellos se pone énfasis en el juego y en la convivencia con otros niños bajo la supervisión de tutores especializados. Los niños apenas se inician en la lectura o en la escritura, ya que la educación es prácticamente lúdica.

En este país se debaten dos tendencias: una, potenciada por los malos resultados obtenidos en el informe PISA, pretende que se ponga mayor

${ }^{1}$ Además de las referencias bibliográficas indicadas, en la elaboración de los apartados comparativos se han utilizado los textos de EURYDICE: 2010 (a), (b), (c) y (d). 
énfasis y se apoye este periodo educativo; y otra, reticente a que la familia pierda el peso específico que aún tiene en la educación de los hijos, que desconfía de los beneficios que pueda aportar una temprana educación formal de los mismos (SCHULTE, 2005: 153).

En España, la educación infantil constituye el primer nivel del sistema educativo y se configura como una etapa educativa con identidad propia que atiende a niños desde su nacimiento hasta los 6 años de edad. A pesar de ser una etapa no obligatoria, posee carácter educativo con un desarrollo estructural y curricular propio. Se organiza en dos ciclos de tres cursos escolares cada uno: el primero hasta los 3 años de edad, y el segundo desde los 3 hasta los 6 años. El segundo ciclo es gratuito, tanto en los centros públicos como en los centros concertados, mientras que las administraciones educativas promueven el incremento progresivo de la oferta de plazas públicas en el primer ciclo, que no tiene carácter gratuito.

En las últimas décadas la educación infantil ha sufrido profundas transformaciones. Si bien es cierto que se está superando la primigenia finalidad asistencial de esta etapa, no lo es menos que hasta hace poco tiempo, en determinadas comunidades autónomas, algunos centros tenían una clara vocación asistencial, llegando incluso a depender de las consejerías de Bienestar Social. No obstante, en ciertos ámbitos sociales aún persisten reminiscencias tendentes a concebir el primer ciclo de educación infantil como asistencial, donde predomina el derecho de padres y madres a su tiempo libre y circunstancias laborales, en claro detrimento del derecho a la educación del niño (SÁNCHEZ MULITERNO, 2009: 57).

En Francia, la educación pre-elemental tiene lugar en la école maternelle, que admite a niños entre 3 y 6 años. Sin ser una etapa obligatoria del sistema escolar francés, es frecuentada por la mayoría de niños desde los 3 años en adelante. Los niños que teniendo 2 años el día del comienzo del curso escolar pueden ser admitidos en las escuelas y las clases maternales, teniendo en cuenta el límite de plazas disponibles y aportando un certificado médico donde se constate el estado de salud y maduración psicológico del niño (SYNDICAT DES ENSEIGNANTS DE FRANCE, 2005: 396). Siendo prioritaria la admisión de los niños de menos de tres años en entornos aislados o en Zonas Prioritarias de Educación (ZEP). Los niños escolarizados durante cuatro años en las escuelas maternales adquieren más conocimientos, vocabulario, escritura, prelectura y numeración (PASSARIEUX, 2009: 19). 
En la escuela maternal existen tres secciones: la básica, para los niños de 2 a 4 años; la media, para los niños de 4 a 5 años; y la grande, para los de 5 a 6 años. Las dos primeras secciones conforman el llamado ciclo de «primeros aprendizajes». Mientras que el siguiente ciclo, el de «aprendizaje fundamental» lo integran la sección grande de la escuela maternal junto con los primeros dos años de la escuela primaria -las clases del curso preparatorio (CP) y el curso elemental (CE1)-. Quedando en evidencia su eminentemente carácter propedéutico y preparatorio para la siguiente etapa de educación elemental, así como la preocupación existente por el tránsito entre la escuela maternal y la educación elemental o primaria.

Los Gobiernos de Inglaterra, Gales e Irlanda del Norte se propusieron y consiguieron, aumentar significativamente la disponibilidad de plazas de educación infantil para los años noventa. En esta década la educación infantil se convirtió en una prioridad de ámbito nacional y local, de modo que las autoridades locales favorecieron su implantación y calidad (ANCHETA ARRABAL, 2007: 227).

En Inglaterra no existe un derecho general a la educación y cuidado de los niños menores de 3 años de edad, aunque los padres pueden optar por pagar a título individual guarderías, cuidadoras o niñeras (privately-run day nursery, childminder o nannies). Para los niños de 3 a 5 años se oferta de manera gratuita una amplia gama de instituciones (públicas, privadas y voluntarias).

Actualmente, en Inglaterra y Gales, los niños de 3 y 4 años tienen derecho a una plaza gratuita en un centro de educación infantil a tiempo parcial. Desde 2004 esta responsabilidad recae sobre las autoridades locales (Ley de 2006 sobre Cuidados de la Infancia), motivo por el que las iniciativas privadas que cumplan los requisitos exigidos son financiadas con fondos públicos.

Ya en 2004, la proporción de alumnos que acudía a centros financiados con fondos públicos entre 3 y 4 años era del 60\%, mientras que el restante $40 \%$ del alumnado acudía a centros privados. Los alumnos con 5 años se encuentran totalmente escolarizados debido a que a esa edad es cuando se inicia la escolarización obligatoria. 


\section{FINALIDAD Y OBJETIVOS}

En virtud de la ley sobre protección de la infancia y la juventud (Kinderund Jugendhilfegesetz) de 1990, en Alemania, los centros de educación infantil tienen la finalidad de fomentar el desarrollo del niño como miembro responsable y autónomo de su comunidad. Éstos desarrollan las labores de instruir, educar y cuidar a los niños, del mismo modo que favorecen su desarrollo social, emocional, físico y mental. La educación y la atención que recibe el alumnado deben ajustarse a la edad de cada niño y al desarrollo de sus capacidades lingüísticas, étnicas,... En definitiva, adaptarse a las necesidades de los niños y sus familias. A tal fin, la educación infantil está diseñada para apoyar y complementar la educación del niño en la familia y para ayudar a los padres a lograr una mejor conciliación laboral y familiar.

Con la finalidad de apoyar y complementar la labor de la familia, la educación infantil pretende el desarrollo personal del alumno, para que se adapte a la vida escolar y se integre adecuadamente en la sociedad, a través del desarrollo de sus facultades físicas y mentales.

A través de las experiencias sensoriales de los alumnos se persigue que sean autónomos en su entorno y que, a raíz de sus propios deseos, ideas y puntos de vista, sean creativos y se conviertan en actores de su propia vida (SCHULGESETZ FÜR DAS LAND BERLIN, 2010).

Desde el acuerdo marco de los Länder para la Educación Temprana en los Centros de Atención Diurna para Niños (Gemeinsamer Rahmen der für die Länder Frühe Bildung en Kindertageseinrichtungen), aprobado por la Conferencia Permanente y la Conferencia de los Ministros de la Juventud en 2004, los objetivos educativos en esta etapa deben enfocarse en las habilidades básicas de comunicación y el fortalecimiento de los recursos personales. Con ellos se pretende motivar al alumnado y prepararlo para asumir y hacer frente a los futuros retos que se encontrará en sus posteriores aprendizajes y en su vida, así como para desempeñar un papel responsable en la sociedad y estar abierto al aprendizaje permanente (EURYDICE, 2010b: 71-72). Concretamente los objetivos que se persiguen se centran en el desarrollo de la personalidad y descubrimiento de las propias habilidades del niño, su autonomía, iniciativa y satisfacción interna por sus propios logros, desarrollar su creatividad, sentidos y su capacidad de entender su entorno, valorar la 
importancia de la vida y de la educación ambiental, teniendo presente que el conocimiento es la base de todas las experiencias de la vida.

En España, la educación infantil constituye la etapa educativa con identidad propia, cuya finalidad es la de contribuir al desarrollo físico, afectivo, social e intelectual de los niños. Respetando la responsabilidad fundamental de las madres y padres o tutores, los centros de educación infantil cooperarán estrechamente con ellos (LOE: Art. 12). Con esta intención se pretende que el alumno conozca su propio cuerpo y el de los otros, que observe y explore su entorno familiar, natural y social, que adquiera autonomía, que desarrolle sus capacidades afectivas, que se relacione con los demás y aprenda a integrarse en la sociedad, que se exprese en diferentes lenguajes ${ }^{2}$, y que se inicie en las habilidades lógico-matemáticas, en la lecto-escritura y en el movimiento, el gesto y el ritmo (LOE: Art. 13). Además se facilitará que los alumnos elaboren una imagen de sí mismos positiva y equilibrada y adquieran autonomía personal (REAL DECRETO 1630/2006: Art. 2.2).

Se pretende conseguir una participación activa de los padres de alumnos en el proyecto educativo del centro y que colaboren en sus actividades, además de mantener una estrecha relación con los educadores, para que unos y otros se puedan transmitir la información que, desde su propia perspectiva, tienen del niño, de sus intereses y necesidades. La educación infantil está vertebrada en dos ciclos que responden ambos a una intencionalidad educativa, no necesariamente escolar, y que obliga a los centros a contar desde el primer ciclo con una propuesta pedagógica concreta. Este ciclo tiene carácter voluntario y su finalidad es la de contribuir al desarrollo equilibrado de las capacidades afectivas, motrices cognitivas y de la comunicación y lenguaje de los niños.

Como fuera que las comunidades autónomas son competentes en este tramo educativo, han procedido a elaborar su propia legislación en la materia. A título de ejemplo señalaremos como, en Andalucía, el primer ciclo tiene entre sus principios generales: Una educación global, integral y personalizada que contribuya al desarrollo de la personalidad, de las capacidades y de las competencias de los alumnos; una equidad en la educación que garantice la igualdad de oportunidades, la no discriminación y la inclusión educativa; y la conciliación entre la vida familiar y laboral de madres y padres (DECRETO 149/2009: Art. 3).

2 Con el Real Decreto 829/2003 se incorporó al currículo una lengua extranjera así como una iniciación en el uso de las nuevas tecnologías de la información. 
En Francia su finalidad radica en ayudar a cada niño a ser autónomo e independiente, a desarrollar sus facultades, a adquirir los conocimientos y las habilidades apropiadas, a desarrollar un lenguaje oral adecuado, bien estructurado y comprensible, y a adentrarse en el mundo de la escritura, los números y demás áreas de aprendizaje. De este modo se pretende alcanzar el objetivo principal, que no es otro que cursar con éxito los primeros años de educación elemental (primaria) (MINISTÈRE DE L'ÉDUCATION NATIONALE, 2012).

En Francia la educación infantil se caracteriza por la minuciosidad con la que ha sido legislada y por su eminente carácter propedéutico, ya que, como hemos indicado, está prevista para preparar al alumno para su posterior ingreso en la escuela elemental. Desde hace tiempo constituye un punto fuerte del sistema educativo francés, sobre todo por su contribución a la mejora de la eficacia de la educación elemental: «El objetivo general de la escuela maternal debe ser el desarrollo de todas las posibilidades del niño, a fin de permitirle formar su personalidad y darle las mejores probabilidades de éxito en la escuela elemental y en la vida, preparándole para los aprendizajes posteriores» (DÉCRET: 6/9/90). Así pues, tiene un doble objetivo, permite que los niños aprendan a vivir en sociedad, a la vez que conforman su personalidad y desarrollan su lenguaje.

En esta etapa el aprendizaje se estructura cinco áreas de conocimientos:

A. Conocimiento de la lengua y de la escritura, para que los niños aprendan a relacionarse y expresarse correctamente, preparándolos para la enseñanza sistemática de la lectura y la escritura que se iniciará en el primer grado de educación elemental.

B. Que sepan discernir y reconocer lo que les distinguen de los demás, a reconocerse como persona, a vivir con otros en una comunidad organizada por normas, a comprender qué es la escuela y cuál es su lugar en ella.

C. Desarrollo de su expresión corporal descubriendo las posibilidades de su cuerpo a través de actividades físicas.

D. Descubrimiento del mundo más cercano, aprendiendo a tomar puntos de referencia espaciales y temporales.

E. Desarrollo sensorial y artístico. 
La finalidad de la educación infantil en Inglaterra radica en el desarrollo de las capacidades sociales, intelectuales, lingüísticas, físicas y cognitivas de los discentes, así como la formación de su personalidad. Si bien, los centros que acogen a los niños más pequeños intentan garantizar su bienestar.

A finales de 1990 las, por entonces, Autoridades de Capacidades y Currículo de Inglaterra (Qualifications and Curriculum Authority) establecieron los objetivos de la educación infantil. Entre ellos se destaca la alfabetización temprana, escritura, aritmética y el desarrollo de habilidades personales y sociales.

El Currículo Nacional establece las siguientes áreas de contenido: desarrollo personal, emocional, físico, social, lingüístico, alfabético, comunicativo y matemático. En los últimos años de la etapa se pondrá especial énfasis en las bases necesarias de lectura, escritura y aritmética, para la implementación de las «tres erres» (wRitting, Reading, aRithmetics) en la posterior reception class.

\section{TIPOS DE CENTROS}

En Alemania, además de los mayoritarios kindergarten, en algunas ciudades también existen los kinderkrippen. Son centros que acogen a niños menores de 3 años, cuya finalidad es esencialmente asistencial. Por otro lado, en algunos centros de educación primaria se imparten unas clases preparatorias (vorklassen), dirigidas a niños de 5 años, cuyos padres quieren que sus hijos reciban una formación preescolar. Pero la asistencia a estas clases actualmente es minoritaria, no llegando a superar el 10\% del alumnado.

Si nos atenemos a su financiación nos hallamos ante dos modalidades de centros, los fundados por organismos privados y los costeados por las propias administraciones locales. En ninguno de los dos casos este servicio es gratuito, los padres deben pagar una serie de mensualidades cuyo coste depende de cada centro. Ambos tipos de establecimientos, que a menudo son confesionales y se caracterizan por ser mixtos, son responsabilidad de las oficinas locales de atención a la juventud que dependen de Asuntos Sociales.

En España, como ya hemos comentado, la educación infantil se estructura en dos ciclos: el primero comprende hasta los 3 años; el segundo, que es gratuito, va desde los 3 a los 6 años de edad. Atendiendo a su financiación los centros pueden ser públicos, privados y privados concertados. 
El primer ciclo se imparte en las escuelas de educación infantil y en los colegios de educación infantil privados. El segundo ciclo depende de las consejerías de educación y se imparte en las escuelas infantiles, y en los centros públicos que ofrecen educación infantil y educación primaria, que se denominan colegios de educación infantil y primaria (CEIP) (LOE: Art. 111)3.

Las administraciones educativas han de velar por la autonomía pedagógica y organizativa de estos centros, favoreciendo el trabajo en equipo del profesorado y su actividad investigadora a partir de la práctica docente. En el ámbito privado existen colegios de distinta denominación.

En el primer ciclo de educación infantil se está favoreciendo el establecimiento de instituciones privadas en detrimento de la educación pública y gratuita, haciendo que este tramo educativo no dependa en gran medida de la Administración educativa pública.

Aunque el segundo ciclo tiene un carácter voluntario, se ha generalizado tanto que en la actualidad prácticamente la totalidad de niños del tramo de edad asisten a instituciones escolares. Una vez alcanzado este logro, las iniciativas ponen el acento en la creación de nuevas plazas educativas para niños de menos de 3 años (MECD: 2012). La mayoría de los centros de este ciclo son de carácter público, no confesionales y mixtos. También existen centros privados autorizados por las administraciones competentes. Los que han optado por la modalidad de centros concertados reúnen similares características que las escuelas públicas, con la salvedad de que pueden ser confesionales. Además, existen instituciones para los niños más pequeños, que no están autorizadas como centros educativos, conocidas habitualmente como «guarderías».

Un caso particular es el de los colegios rurales agrupados que se encuentran en las zonas rurales del país. Se trata de agrupaciones de varios centros que están dispersos y conforman una sola institución. Imparten educación infantil y primaria desde los 3 hasta los 12 años de edad, son públicos y de carácter no confesional.

En Francia los centros de educación infantil pueden depender bien del Ministerio de Educación Nacional, como es el caso de las escuelas

3 La normativa sobre educación infantil está enmarcada en la LOE y en el Real Decreto 1630/06 de 29 de diciembre por el que se regulan las enseñanzas mínimas del segundo ciclo de infantil en todo el Estado, quedando completada con los decretos que sobre educación infantil han aprobado las distintas comunidades autónomas. 
maternales (écoles maternelles) y las secciones de educación infantil de las escuelas elementales; bien del Ministerio de Salud y Seguridad Social, como los asisstantes maternelles, los créches collectives o familiales las pouponniers y las haltes garderies.

La escuela maternal ofrece educación infantil a niños entre los 2 y los 6 años. Sus estudios están coordinados con la etapa de educación primaria. Sin ser una etapa obligatoria del sistema escolar francés, es frecuentada por la inmensa mayoría de niños desde la edad de 3 años en adelante. El Ministerio de Educación es el responsable último de estas instituciones y, por tanto, el encargado de establecer el plan de estudios, la formación y contratación de los docentes y su mantenimiento en general.

Como rasgos característicos de las escuelas maternales públicas destacaremos que son, laicas y gratuitas, están financiadas por los municipios y forman parte de un sistema educativo centralizado, lo que garantiza su presencia en todo el territorio y los mismos objetivos de aprendizaje. Mientras que los privados generalmente son confesionales, y al igual que los centros públicos son mixtos.

A veces, cuando la distancia que ha de recorrer el transporte escolar o los gastos a realizar son excesivos, se crean en las propias escuelas de educación elemental secciones infantiles, destinadas a acoger a niños que ya han cumplido los 5 años de edad, conformándose éste como uno de los primeros elementos de desigualdad escolar.

En Inglaterra existe una variada oferta de centros de educación infantil, que pasamos a agrupar:

A. Nursery classes, Nursery schools and Day nurseries.

Las nursery classes y las nursery schools pueden ser estatales o privadas, mientras que las day nurseries son mayoritariamente privadas.

La mayor parte de las nurseries atienden a niños entre 3 y 5 años, aunque muchas day nurseries aceptan niños de menor edad. Están abiertas durante el curso escolar, aunque muchas day nurseries también tienen horarios durante las vacaciones escolares. Ofrecen 5 sesiones semanales de medio día, sin embargo, algunas ofrecen jornadas de tiempo parcial y tiempo completo en función de las necesidades de los padres.

Las nursery schools y classes tienen una ratio de 20 a 26 alumnos por cada dos profesionales, uno de los cuales debe ser maestro y el otro asistente 
de guardería cualificado. Las day nurseries tienen más personal, y sus normas, reglas y cualificaciones del personal difieren en función de las edades de los niños que atienden.

\section{B. Centros para niños Sure Start.}

Son centros infantiles que ofrecen una gran variedad de servicios para padres e hijos. Sus prestaciones están disponibles desde el embarazo hasta los 5 años, edad a la que los niños acceden a las reception classes. Mientras los niños aprenden, juegan y están cuidados, los padres pueden beneficiarse de una serie de prestaciones, que varían en función de las necesidades de cada localidad. A modo de ejemplo citamos las siguientes:

- Asistencia sanitaria para el niño y la familia, que incluye desde servicios de visita médica hasta apoyo a la lactancia materna.

- Cuidados infantiles de alta calidad y aprendizaje temprano.

- Clases para padres y acceso a servicios especializados para familias como terapias sobre logopedia, consejos sobre alimentación sana y asesoramiento jurídico.

- Orientación para la búsqueda de trabajo o estudios de formación laboral.

La atención a los menores es gratuita para niños entre 3 y 4 años. Para los demás sí han de ser abonados, sin menoscabo de la existencia de ayudas para sufragar los gastos.

\section{Preschool Playgroups.}

Los grupos de juego son en su mayoría instituciones sin ánimo de lucro. Pueden estar gestionados por voluntarios, incluso por los propios padres, y proporcionan cuidados y atención temprana a los menores de 5 años. Aunque existe una enorme variedad y tipos de playgroups, la mayoría de ellos tienen las siguientes características:

- Atienden a niños entre 3 y 5 años, aunque en algunos casos se amplía hasta los 2 años.

- Están abiertos durante todo el año escolar.

- Generalmente ofrecen sesiones de medio día, aunque algunos pueden ofrecer más horas.

- En su mayoría atienden entre 10 y 20 niños, siempre con la presencia de un adulto por cada ocho niños. Al menos, la mitad de los cuidadores deben ser personal cualificado. 


\section{Reception Classes.}

Algunas escuelas primarias pueden llegar a admitir a niños menores de 5 años en estas aulas de acogida. Se encuentran abiertas durante todo el curso escolar. Al principio los niños suelen asistir sólo en sesiones de media jornada, hasta completar progresivamente la jornada a tiempo completo. Se encuentran limitadas por ley a un máximo de 30 alumnos y la mayoría cuentan con asistentes.

\section{E. Childminders.}

Se trata de niñeras autorizadas que llevan a cabo este servicio, por lo general, en su propia casa. En aquellos casos en los que se ofrece este servicios de manera gratuita para los niños de 3 y 4 años de edad, deben estar registrados por el Office for Standards in Education, Children's Services and Skills (OFSTED) y acreditados por las autoridades locales.

Todas las niñeras deben cumplir ciertos estándares de calidad en sus instalaciones y deben estar controladas e inspeccionadas regularmente para garantizar que tanto el lugar como el personal son aptos para el cuidado de los menores.

Este servicio está dirigido para niños desde unos meses de vida hasta los 5 años. A menudo suelen tener flexibilidad de jornada laboral y horario, que dependerá de cada caso en particular. Algunas se encargan del cuidado de los niños después de su horario escolar o en los días festivos. Pueden llegar a cuidar un máximo de seis niños de hasta 8 años de edad, aunque no más de tres deben ser menores de 5 años.

Tabla 1. Instituciones educativas y edades de la educación infantil en la UE

\begin{tabular}{|c|c|c|c|c|}
\hline \multirow{2}{*}{ PAÍS } & \multirow{2}{*}{ EDAD } & \multirow{2}{*}{ DENOMINACIÓN } & \multicolumn{2}{|c|}{ ANTECEDENTES ${ }^{4}$} \\
\hline & & & s. XVIII & s. XIX \\
\hline \multirow{3}{*}{ ALEMANIA } & 0 a 3 años & Kinderkrippen & \multirow{3}{*}{$\begin{array}{l}\text { Asilos de } \\
\text { niños }\end{array}$} & \multirow{3}{*}{ Kindergarten } \\
\hline & 3 a 6 años & Kindergarten & & \\
\hline & 5 años & Vorklassen & & \\
\hline \multirow{3}{*}{ ESPAÑA } & 1 a 6 años & Escuelas de Educación Infantil (2 ciclos) & \multirow{3}{*}{$\begin{array}{c}\text { Escuelas } \\
\text { amigas }\end{array}$} & \multirow{3}{*}{$\begin{array}{l}\text { Escuelas de } \\
\text { Párvulos }\end{array}$} \\
\hline & 1 a 6 años & Centros Concertados de Educación Infantil & & \\
\hline & 3 a 6 años & $\begin{array}{l}\text { Colegios Rurales Agrupados (imparten educación } \\
\text { inicial y primaria) }\end{array}$ & & \\
\hline
\end{tabular}




\begin{tabular}{|c|c|c|c|c|}
\hline \multirow{2}{*}{ PAÍS } & \multirow{2}{*}{ EDAD } & \multirow{2}{*}{ DENOMINACIÓN } & \multicolumn{2}{|c|}{ ANTECEDENTES ${ }^{4}$} \\
\hline & & & s. XVIII & s. XIX \\
\hline \multirow{5}{*}{ FRANCIA } & 2 a 6 años & École Maternelle & \multirow{5}{*}{$\begin{array}{l}\text { Petites } \\
\text { écoles à } \\
\text { tricoter } \\
\text { Poêles à } \\
\text { tricoter }\end{array}$} & \multirow{5}{*}{$\begin{array}{c}\text { Salas de asilo } \\
\text { Escuelas } \\
\text { maternales }\end{array}$} \\
\hline & 5 a 6 años & Sección Infantil de las Escuelas Elementales & & \\
\hline & $\begin{array}{l}2 \text { meses } \\
\text { a } 3 \text { años }\end{array}$ & Créches & & \\
\hline & 3 a 6 años & Haltes garderies & & \\
\hline & $\begin{array}{l}18 \text { meses } \\
\text { a } 3 \text { años }\end{array}$ & Pouponniers & & \\
\hline \multirow{4}{*}{ INGLATERRA } & $\begin{array}{l}18 \text { meses } \\
\text { y } 5 \text { años }\end{array}$ & Nursery Center (Inglaterra y P. Gales) & \multirow{4}{*}{$\begin{array}{c}\text { Dame } \\
\text { schools }\end{array}$} & \multirow{4}{*}{$\begin{array}{l}\text { Escuelas } \\
\text { infantiles }\end{array}$} \\
\hline & $\begin{array}{l}21 / 2 \text { a } 5 \\
\text { años }\end{array}$ & Pre-school Group (Inglaterra, P. Gales e Irlanda) & & \\
\hline & 3 a 5 años & Nursery School (Inglaterra, P. Gales e Irlanda) & & \\
\hline & $\begin{array}{l}\text { Menores } \\
\text { de } 5 \text { años }\end{array}$ & Day Nursery (Inglaterra, P. Gales e Irlanda) & & \\
\hline
\end{tabular}

Fuente: Elaboración propia.

\section{EDAD, AGRUPACIONES Y RATIOS}

La educación obligatoria comienza en la mayoría de los países de la UE en el nivel primario. En los países estudiados, la edad de inicio de la educación primaria obligatoria es mayoritariamente los 6 años, salvo en Inglaterra que comienza a los 5 años, integrándose los alumnos directamente en programas de educación primaria.

Sin embargo, los niños comienzan su educación formal a una edad cada vez más temprana. Durante el período de 2000 a 2009, las tasas de participación media de la UE-27 aumentaron un 15,3\% en el caso de los niños de 3 años de edad, un 7\% en los 4 años y un 6,3\% más en el de los menores de 5 años. Llegando a alcanzar respectivamente casi un 77\%, 90\% y $94 \%$ en 2009. Para este año, la participación de los niños de 3 años en el segundo ciclo de educación infantil era casi completa en España y Francia, llegando a más del 95\% (EURYDICE, 2012: 68).

4 García Garrido (1989); Sanchidrián y Ruiz Berrio (2010). 
Como puede observarse en la tabla 2, el segundo ciclo de educación infantil se conforma como un periodo significativo del sistema escolar. En los casos de Francia y España los porcentajes de alumnado matriculado en este ciclo son bastante elevados, mientras que en Alemania, son algo inferiores. En el Reino Unido, aunque los datos son bastante más bajos, debemos recordar que la educación obligatoria comienza un año antes que en el resto de países, por lo que en la proporción reflejada no se tiene en cuenta los niños de 5 años.

Tabla 2. Alumnos inscritos en segundo ciclo de Educación Infantil (2009)

\begin{tabular}{|l|c|c|c|c|}
\cline { 2 - 5 } \multicolumn{1}{c|}{} & $\begin{array}{c}\text { EDAD DE } \\
\text { FINALIZACIÓN } \\
\text { DEL CICLO }\end{array}$ & $\begin{array}{c}\text { \% DE ALUMNOS } \\
\text { CON RESPECTO } \\
\text { AL TOTAL } \\
\text { DE TODOS } \\
\text { ESTUDIANTES }\end{array}$ & $\begin{array}{c}\text { \% GRUPO DE } \\
\text { TOTAL } \\
\text { (EN MILES) }\end{array}$ & $\begin{array}{c}\text { AÑD ENTRE 4 } \\
\text { ESCOLARIDAD } \\
\text { OBLIGATORIA }\end{array}$ \\
\hline ALEMANIA & 6 & 14,6 & $2.385,9$ & 96 \\
\hline ESPANA & 6 & 18,7 & $1.765,6$ & 99,3 \\
\hline FRANCIA & 6 & 17,3 & $2.554,1$ & 100 \\
\hline REINO UNIDO & 5 (Inglaterra) & 8,1 & $1.121,7$ & 97,3 \\
\hline
\end{tabular}

Fuente: EUROSTAT, Education and Training.

Se pueden distinguir, principalmente, dos modelos de centros que acogen a niños antes de que ingresen en el sistema escolar obligatorio. Los alumnos se agrupan bien atendiendo al «modelo escuela», que es el más común, bien al «modelo de familia». El primero viene a ser como un «anticipo» de la escuela primaria, donde los niños son agrupados por edades. El segundo se conforma como un evocador de la familia asemejándose a la situación familiar y, en este caso, los niños de distintas edades se reúnen en un mismo grupo.

Sin embargo, no todos los países prescriben que las escuelas deben decantarse por un modelo determinado. En algunos países, la decisión se deja en mano de las autoridades locales educación responsable de los centros, como ocurre en Inglaterra donde los playgropups adoptan el «modelo de familia» aunque el «modelo escuela» sea el más frecuente. En general, los países optan por el modelo de escuela, tales son los casos de Francia, España y algunos centros ingleses, así como de las zonas rurales donde no viven suficientes alumnos para establecer un único grupo por edad (v.g.: en ciertas regiones de España). Sólo en unos pocos países prevalece el modelo de familia, como ocurre en Alemania. 
La gran mayoría de países de la UE han introducido normas para fijar una ratio máxima de alumnos en las instituciones de segundo ciclo de educación infantil. Si se excede dicho límite, debe dividirse el grupo o contar con un mayor número de cuidadores. Casi dos tercios de los países de la UE cuentan con un límite entre 20 y 25 niños, entre los que se encuentran Alemania y España. El resto de los países, entre los que se encuentra Inglaterra, prevén grupos inferiores a 15 alumnos.

Tabla 3. Número máximo de niños recomendado por cada adulto cualificado en segundo ciclo de educación infantil. Curso 2010/11

\begin{tabular}{|l|c|c|c|}
\cline { 2 - 4 } \multicolumn{1}{c|}{} & \multicolumn{3}{c|}{ EDAD } \\
\cline { 2 - 4 } \multicolumn{1}{c|}{} & 3 años & 4 años & 5 años \\
\hline ALEMANIA & 25 & 25 & 25 \\
\hline ESPAÑA & 25 & 25 & 25 \\
\hline FRANCIA & Sin regulaciones & Sin regulaciones & Sin regulaciones \\
\hline INGLATERRA & 13 & 13 & Sin regulaciones \\
\hline
\end{tabular}

Fuente: EURYDICE, 2012: 152.

La agrupación por edades es la forma más usual en la educación temprana y cuidados infantiles. En todos los países europeos, existe alguna forma de atención temprana y cuidado (Early childhood education and care -ECEC-) subvencionada públicamente y acreditada, estando destinada a niños que no han alcanzado la edad de escolaridad obligatoria.

Dos son los modelos de organización principales de los servicios de ECEC en Europa: los de una sola fase y los de fase dual. Sin menoscabo de que en algunos países se combinan ambos modelos. En el marco del primer modelo, la provisión para los niños pequeños se desarrolla de forma unitaria, organizada en una sola fase para todos los niños que no han alcanzado la edad de escolarización obligatoria. Cada establecimiento tiene un solo equipo de gestión para los niños de todas las edades y, generalmente, el personal responsable de la educación de los niños tiene las mismas cualificaciones y similares escalas salariales, independientemente de la edad de los niños que se encuentren a su cargo. 
En España, la configuración de centros unitarios, que atienden a los niños hasta los 3 años, existe junto con la configuración de doble fase. La mayoría de países europeos, sin embargo, sigue el segundo modelo, de fase dual, donde la atención temprana y cuidados financiados con fondos públicos se divide de acuerdo a la edad de los niños. El organismo responsable de la formulación de políticas y ejecución de la prestación también suele diferir entre las etapas. Los niños con edades comprendidas entre los 3 y 6 años suelen integrarse en las estructuras que forman parte del sistema nacional de educación. Este es el caso de Alemania y Francia. Incluso en Francia, hasta los niños con 2 años de edad pueden llegar a integrarse en el sistema escolar general.

Independientemente del modelo de la ECEC adoptado, en muchos países la prestación para los niños más pequeños (hasta los 3 años) está sujeta a variaciones locales. A menudo, las autoridades locales son las únicas responsable para decidir cómo organizar los servicios subvencionados. Este es el caso de casi todos países del centro y este de Europa. En Inglaterra, las plazas financiadas y gratuitas en su totalidad están disponibles para todos los niños mayores de 3 años y para algunos niños desfavorecidos mayores de 2 años. Ante la inexistencia del derecho a una educación inicial y cuidado de los niños menores de 3 años, los padres pueden optar por pagar un servicio de guardería para estos niños.

Tabla 4. Cuidados Formales ${ }^{5}$ de la Infancia por duración y grupos de edad. Porcentaje de niños atendidos respecto al total de niños de su misma edad (2009)

\begin{tabular}{|l|c|c|c|c|c|c|}
\cline { 2 - 7 } \multicolumn{1}{c|}{} & \multicolumn{4}{c|}{ MENORES DE 3 AÑOS (\%) } & \multicolumn{3}{c|}{$\begin{array}{c}\text { ENTRE 3 AÑOS Y EDAD DE EDUCA- } \\
\text { CIÓN OBLIGATORIA (\%) }\end{array}$} \\
\cline { 2 - 7 } & $\begin{array}{c}\text { Sin Cuidados } \\
\text { Formales }\end{array}$ & $\begin{array}{c}\text { Entre 1 y 29 } \\
\text { horas/semana }\end{array}$ & $\begin{array}{c}\text { 30 horas o } \\
\text { más / semana }\end{array}$ & $\begin{array}{c}\text { Sin Cuidados } \\
\text { Formales }\end{array}$ & $\begin{array}{c}\text { Entre 1 y 29 } \\
\text { horas/semana }\end{array}$ & $\begin{array}{c}30 \text { horas o } \\
\text { más / semana }\end{array}$ \\
\hline ALEMANIA & 81 & 7 & 12 & 11 & 48 & 40 \\
\hline ESPAÑA & 59 & 18 & 18 & 6 & 50 & 44 \\
\hline FRANCIA & 64 & 16 & 25 & 5 & 48 & 47 \\
\hline REINO UNIDO & 65 & 31 & 4 & 9 & 70 & 21 \\
\hline
\end{tabular}

Fuente: EUROSTAT, Income and Living Conditions

5 Como cuidados formales se consideran cuatro tipos de servicios: la educación preescolar, la educación obligatoria en la escuela, cuidado de niños en instituciones fuera del horario escolar (antes / después) y guardería. Por lo tanto, se incluyen todo tipo de cuidados organizado / 


\section{FINANCIACIÓN}

La creciente demanda de este tipo de centros en los países de la UE ha provocado que las distintas administraciones faciliten el acceso a los mismos La educación infantil no obligatoria se oferta cada vez más de manera gratuita, siendo accesible a las familias de bajos ingresos económicos. La financiación privada del segundo ciclo de educación infantil tiende a disminuir, dadas las facilidades que ofrecen los centros escolares públicos. Incluso se suelen ajustar las cuotas a pagar en función de los ingresos familiares y otros criterios.

En algunos países, las instituciones cobran tasas para determinados grupos de edad, frecuentemente para los alumnos que tienen menos de 3 años. En el caso de España, los padres pagan durante el primer ciclo de educación infantil, pero no en los siguientes. En Francia, la prestaciones del sector público son gratuitas, aunque algunos servicios adicionales como el comedor o el transporte deben ser abonados. Si bien, en el sector privado sí se pagan tasas de matrícula. En el Reino Unido, se ofertan instituciones de manera gratuita a tiempo parcial para los niños de 3 y 4 años, soliendo ofrecer horas extras, en este caso, de pago. En esta etapa, el sector privado está muy extendido, siendo los padres del Reino Unido quienes pagan los más altos costes por la educación y el cuidado de sus hijos de toda Europa (LYNDON, 2000: 184, citado por ANCHETA ARRABAL, 2007: 228).

Tabla 5. Oferta de gratuidad y pago de las instituciones de educación infantil. Curso 2010/11

\begin{tabular}{|l|l|l|}
\cline { 2 - 3 } \multicolumn{1}{c|}{} & \multicolumn{1}{c|}{ INSTITUCIONES PÚBLICAS } & \multicolumn{1}{c|}{$\begin{array}{c}\text { INSTITUCIONES PRIVADAS } \\
\text { SUBVENCIONADAS }\end{array}$} \\
\hline ALEMANIA & Abono de tasas & Abono de tasas \\
\hline ESPAÑA & Gratuita & Gratuita \\
\hline FRANCIA & Gratuita & Abono de tasas \\
\hline INGLATERRA & Gratuita & Gratuitas a tiempo parcial \\
\hline
\end{tabular}

En la Unión Europea, además de las políticas generales destinadas a combatir la exclusión social, en todos los países donde esta etapa no es

controlado por una estructura (pública, privada). La atención recibida por cuidadores sin ningún tipo de estructura entre el cuidador y los padres (acuerdos directos) ha sido excluida con el fin de tener en cuenta de cuidado de niños sólo reconocida como el cumplimiento de ciertos patrones de calidad. La duración se refiere al número promedio de horas durante una semana normal. 
gratuita se prevén ayudas para sufragar sus gastos, en función de la situación socioeconómica de los padres. Mayoritariamente los mecanismos de apoyo se establecen a nivel central y es el ingreso familiar el factor determinante. Además, con cierta frecuencia, se tienen en cuenta otros criterios: como el número de hijos y la situación civil-familiar (ej.: los niños que viven en hogares monoparentales). En el Reino Unido existen créditos fiscales para las familias que se encuentren por debajo de un determinado nivel de ingresos y tienen que pagar las horas extraordinarias no cubiertas por fondos públicos. En Alemania se aplican tarifas más bajas en áreas geográficas específicas.

Tabla 6. Factores que se tienen en cuentas en la oferta de reducciones o exenciones de tasas en instituciones de educación infantil públicas o privadas subvencionadas. Curso 2010/11

\begin{tabular}{|l|c|c|c|c|c|}
\cline { 2 - 6 } \multicolumn{1}{c|}{} & $\begin{array}{c}\text { INGRESOS } \\
\text { FAMILIARES }\end{array}$ & $\begin{array}{c}\mathrm{N}^{\circ} \mathrm{DE} \\
\text { HIJOS }\end{array}$ & $\begin{array}{c}\text { ESTADO CIVIL (FAMILIAS } \\
\text { MONOPARENTALES) }\end{array}$ & $\begin{array}{c}\text { UBICACIÓN } \\
\text { GEOGRÁFICA }\end{array}$ & $\begin{array}{c}\text { SIN } \\
\text { GASTOS }\end{array}$ \\
\hline ALEMANIA & $\mathrm{X}$ & $\mathrm{X}$ & $\mathrm{X}$ & $\mathrm{X}$ & \\
\hline ESPANAA & & & & & $\mathrm{X}$ \\
\hline FRANCIA & $\mathrm{X}$ & & & & \\
\hline INGLATERRA & $\mathrm{X}$ & & & & \\
\hline
\end{tabular}

Fuente: EURYDICE, 2012: 98.

\section{PROFESORADO}

Con la intención de conseguir un cuerpo de profesores de calidad y bien formado, en todos los países de la UE se les exige una formación académica que incluya conocimientos teóricos y prácticos. Los distintos países se han decantado por uno de los siguientes dos modelos de formación inicial del profesorado: A. El modelo simultáneo, que integra a la vez su formación pedagógica como profesional de la educación y el estudio de las materias que como docente tendrá que enseñar en un futuro. B. El modelo consecutivo, los estudiantes adquieren primero una formación académica más general y posteriormente cursan una enseñanza profesional de carácter pedagógico, completando su grado académico.

Desde el inicio del proceso de Bolonia, la formación inicial del profesorado ha sufrido muchos cambios. En casi todos los países de la UE, los maestros de los niveles de infantil y primaria lo hacen bajo el modelo 
simultáneo, tal es el caso de España y Alemania. En Francia se lleva a cabo el modelo consecutivo. Aunque en Inglaterra, es más habitual éste último, persisten ambos modelos.

Respecto al nivel de formación que los docentes deben alcanzar para convertirse en maestros cualificados existen diferencias entre unos y otros países. En la mayoría de ellos se exige el título de licenciado, de grado o uno equivalente, que permita ejercer como maestro de educación infantil, tales son los casos de España e Inglaterra, que cuentan con una serie de particularidades que comentamos a continuación. En España, los profesores que deseen impartir clases en el primer ciclo deben tener la titulación de Maestro especialista en Educación Infantil o titulo de Técnico Superior de Educación Infantil. Mientras que el título necesario para impartir clases al Segundo Ciclo, es el de Maestro especialista en Educación Infantil, pudiendo ser apoyados, en su labor docente, por maestros de otras especialidades cuando las enseñanzas impartidas lo exijan (LOE: Art. 92). En Inglaterra, se requiere además superar el ITE (Inicial Teacher Education), fase de apoyo a los profesores recién titulados al comienzo de su primera enseñanza. Durante este periodo llevan a cabo tareas relacionadas con la práctica y son remunerados por su trabajo.

En Alemania, el nivel mínimo de formación inicial de los docentes es el de secundaria superior o post-secundaria no terciaria. Por el contrario, en el caso de Francia se requiere el grado de master y reciben la misma formación que los de primaria, por tanto, pueden enseñar en todos los grados de primaria.

En general, cabe señalar que el nivel mínimo de formación necesaria para convertirse en un maestro de educación infantil ha aumentado en Europa en comparación con el curso 2006/07 (Eurydice, 2012).

Tabla 7. Formación requerida y extensión mínima de formación inicial para profesores de educación infantil. Curso 2010/11

\begin{tabular}{|l|l|l|}
\cline { 2 - 3 } \multicolumn{1}{c|}{} & \multicolumn{1}{c|}{ DURACIÓN } & \multicolumn{1}{c|}{ TITULACIÓN } \\
\hline ALEMANIA & 3 años & Secundaria Superior / Postsecundaria no terciaria \\
\hline ESPAÑA & 4 años & Grado (Bachelor's) \\
\hline FRANCIA & 5 años & Máster \\
\hline INGLATERRA & $4+1$ años & Grado (Bachelor's) + ITE \\
\hline
\end{tabular}

Fuente: EURYDICE, 2012: 112. 


\section{APORTACIONES Y REFLEXIONES FINALES}

La educación infantil implantada en Alemania, Francia, España e Inglaterra cuenta con dos características que se compaginan de distinta forma, nos referimos a su objetivo asistencial y a su finalidad educativa. En este sentido, propugnamos que los centros de educación infantil, en todos sus ciclos, dependan de los Ministerios, Departamentos y/o Consejerías de Educación, y no de Asuntos Sociales o Bienestar Social, con la meridiana intención de conseguir que prevalezca la finalidad educativa sobre la asistencial.

En esta etapa, donde los aprendizajes tempranos adquieren gran relieve como potenciadores de la estimulación y desarrollo de múltiples habilidades cognitivas, las necesidades laborales han influido decisivamente en la cada vez mayor delegación de funciones otrora propias de la familia en las instituciones escolares, perdiendo ésta gran parte de su papel educativo. En ella se ha de involucrar no sólo al niño sino a los padres de familia. Por este motivo, las instancias educativas realizan esfuerzos por vincular el ambiente natural del niño, es decir, la familia, con el ambiente educativo (ORGANIZACIÓN DE LOS ESTADOS AMERICANOS, 2009: 5).

Como respuesta a una creciente demanda, en los cuatro países estudiados se ha producido un fuerte incremento de las instituciones que se dedican a la educación infantil, pasando a realizar un importante papel de apoyo a las familias. Entre las causas que influyen en esta demanda se encuentran los profundos cambios que están experimentando las familias y las crecientes necesidades y deseos de la sociedad capitalista actualmente imperante en Europa. Circunstancias tales como la progresiva incorporación de la mujer al mundo laboral, las distancias entre hogares y centros de trabajo, el aumento de las familias monoparentales, desestructuradas... hacen aún más necesario que el menor esté rodeado de un grupo de iguales que favorezcan su proceso de socialización. Circunstancia que ha propiciado que la educación infantil vaya más allá de una mera función educativa al pretender dar respuesta a necesidades familiares y laborales.

Estimamos que se debe evitar la presión social que propicia que los alumnos reciban de forma temprana una educación formal estructurada en materias, adelantado de forma excesiva objetivos y contenidos escolares, como apuntan países como Francia, España e Inglaterra. Sino que, prioritariamente, se les debe fomentar las ganas de aprender, ayudar a construir sus destrezas 
sociales, su lenguaje y su confianza a través del juego y teniendo en cuenta sus intereses y desarrollo, tal y como tradicionalmente se viene haciendo en los kindergarten alemanes.

Desde un punto de vista psicopedagógico, se preconiza la necesidad de un modelo de escuela infantil que responda a las diferentes etapas evolutivas del niño y que favorezca el desarrollo integral del mismo, dirigido por especialistas que garanticen la participación de los padres. La Educación Infantil puede y debe favorecer, de manera eficaz, la compensación de numerosas desigualdades, especialmente las carencias originadas por deficiencias relativas al entorno social, cultural y económico, sin que ello, en ningún momento, signifique dejar de reconocer las diferencias psicológicas de los discentes, que por supuesto, no sólo han de ser respetadas, sino además atendidas. La progresiva generalización de esta primera etapa educativa está favoreciendo la disminución de las desigualdades sociales en pro de una mayor igualdad de oportunidades de los niños.

No existe uniformidad en cuanto a la asistencia de los niños menores de 3 años a los centros de educación preescolar, ya que los posicionamientos ideológicos de cada familia y sus distintas sensibilidades tienen un fuerte peso específico en cuanto a la edad más propicia para ingresar por primera vez al menor en un centro educativo. En este sentido, constatamos como muchas familias alemanas se resisten a inscribir a sus hijos en los centros de primer ciclo de educación infantil, mientras que en España estas instituciones cuentan con una fuerte demanda, y en Inglaterra han optado por una gran diversidad de posibilidades.

Nos cuestionamos la actual tendencia a escolarizar indiscriminadamente a los niños menores de 3 años. Estimamos que no se debe forzar la admisión indistinta de niños que aún no poseen autonomía para satisfacer sus propias «necesidades primarias» (aseo, comida, desplazamientos, vestirse,...), que no se han «individualizado» y que todavía no han adquirido la capacidad de hablar de sí mismo y de expresarse en primera persona. La finalidad educativa de la educación infantil constituye un derecho de todos los niños que adquieren desde su nacimiento hasta los 6 años de edad y, como tal, se ha de incorporar al sistema educativo de cada país. Si bien, estimamos no debe adquirir el carácter de escolarización obligatoria. 
Si tenemos en cuenta el nivel de desarrollo de estos niños, quizás el simple aumento de este tipo de centros no sea la solución más adecuada. Más bien se podría buscar otro tipo de vías más personalizadas que dieran respuesta, por un lado, a las demandas sociales en pro de una atención temprana de los hijos por motivos laborales y para prepararlos ante su inminente ingreso en centros de educación preescolar; mientras que, por otro lado, se debe favorecer y potenciar una mayor relación de los hijos con sus padres. El fortalecimiento del factor educativo alejaría el riesgo de reducir estos centros de educación preescolar a simples guarderías.

El currículo de Educación Infantil en Inglaterra gira en torno a la formación de los hábitos de autonomía personal, los centros del interés del alumnado, el aprendizaje por descubrimiento con una gran influencia de las teorías constructivistas aplicadas al aprendizaje, la psicomotricidad y las actividades manuales y manipulativas. En España la enseñanza ha de presentarse de forma globalizada teniendo presente las características individuales de los alumnos. A los que se les ayudará a construir su propia identidad, desarrollando su autonomía, su autoestima y un equilibrio emocional adecuado a través de la adquisición de valores como respetar a los demás y sus trabajos, aceptar las normas de convivencia, respetar el medio ambiente $^{6}, \ldots$ En Alemania se utilizan estrategias compensatorias matriculando a los niños que no están preparados para el primer grado, en clases de primaria, pero con una orientación pedagógica similar a la del preescolar. En Francia utilizan una metodología basada en la pedagogía del juego, la investigación y la acción, que permita las experiencias sensoriales, motrices, afectivas e intelectuales de los alumnos. Al igual que en España los centros han de elaborar su propio Proyecto de Escuela.

Los niños, como el resto de las personas transitan a lo largo de su vida por distintas etapas. Esta transición ha de ser entendida como un proceso continuo donde todas las acciones que se realizan, antes, durante, y después son relevantes. Así pues, esta unidad de la etapa debe implicar que la división de la educación infantil en dos ciclos no ha de suponer diferencias esenciales entre sus respectivas finalidades, planteamientos y requisitos.

6 Real Decreto 114/2004, de 23 de enero, por el que se establece el currículo de la Educación Infantil. 
La educación infantil es una etapa única, con características propias y con entidad educativa por sí misma ${ }^{7}$, constituyendo la primera etapa de cada sistema educativo, que no debe ser considerada exclusivamente como preparatoria de etapas posteriores, aunque ésta sea una de sus funciones. Pero como, por otro lado, el desarrollo del niño es un proceso continuo en el que no es fácil delimitar momentos de clara diferenciación y ruptura, y porque los cambios no se producen de modo uniforme en todos los niños. Esta etapa ha de estar en estrecha coordinación con las instituciones de Educación Primaria, para garantizar así un tránsito adecuado del alumno. En este sentido, hemos de añadir que en los cuatro países analizados se realiza una la fundamentación del currículo para facilitar la preparación del discente en su posterior paso por la escuela.

Una manera de facilitar esta transición es articular los currículos de los distintos niveles, no sólo en los contenidos, sino también en los métodos de enseñanza y aprendizaje. En algunos países como España y Francia se han articulado los currículos del nivel preescolar con los de la escuela primaria o los currículos de los centros de cuidado y educación con el de preescolar, bajo un solo marco curricular para la primera infancia, como es el caso de Inglaterra, donde además, se realiza una articulación curricular y se establecen alianzas entre quienes trabajan en el nivel preescolar y la escuela primaria. En España se pone énfasis en la descentralización y la autonomía de los centros en la aplicación del currículum, de manera que éste pueda responder a los diversos contextos sociales y culturales del país, mientras que en Inglaterra y Alemania ésta es la norma. En Inglaterra y Francia, cuando los niños entran a la escuela tienen marcados unos objetivos fijos y cuentan con un sistema de evaluación.

A nivel de la UE se están haciendo esfuerzos para alcanzar cierto grado de convergencia. Se coincide en que la educación infantil y la atención a la infancia han de responder al doble desafío de ofrecer un acceso equitativo generalizado a todos, y aumentar al mismo tiempo su calidad. Para conseguirlo el Consejo de la Unión Europea ha propuesto una serie de medidas, entre las que se encuentran las siguientes ${ }^{8}$ :

1. Facilitar el acceso equitativo a una educación infantil y una atención a la infancia de alta calidad, en particular a los niños que proceden de un entorno socioeconómico desfavorecido o con necesidades educativas especiales.

\footnotetext{
7 «La educación infantil constituye una etapa educativa con identidad propia» (REAL DECRETO 1630/2006, 474).

8 Consejo de la Unión Europea (2011: 1).
} 
2. Concebir modelos de financiación eficaces.

3. Fomentar enfoques intersectoriales e integrados para los servicios de educación, una transición fluida entre los diferentes niveles de educación.

4. Apoyar la profesionalización del personal.

5. Promover programas y planes de estudio adecuados.

6. Apoyar a los padres en su papel de principales educadores de sus hijos.

7. Promover la garantía de la calidad con la participación de las principales partes interesadas, incluidas las familias.

Aunque exista cierto consenso en las medidas a adoptar a nivel de la UE, no es menos cierto que las políticas relativas a la educación infantil imperantes en cada país estudiado se basan en enfoques claramente diferenciados. Su historia, contextos, valores, creencias y situación socioeconómica inciden fuertemente en las decisiones que cada uno de ellos ha adoptado (OCDE, 2001: 3).

\section{REFERENCIAS BIBLIOGRÁFICAS}

ANCHETA ARRABAL, A. (2007): La formación inicial del profesorado de educación infantil en Italia, Reino Unido y España: una perspectiva comparada, Revista Española de Educación Comparada, 13, pp. 219-251.

ASOCIACIÓN DE MAESTROS ROSA SENSAT (2004): El papel de Europa en la educación infantil, Infancia en Europa, 7.

CONSEJO DE LA UNIÓN EUROPEA: Conclusiones del Consejo sobre educación infantil y atención a la infancia: ofrecer a todos los niños la mejor preparación para el mundo de mañana 2011/C 175/03. C 175/8. Diario Oficial de la Unión Europea 15.6.2011.

DÉCRET no 90-788 du 6 septembre 1990. Organisation et fonctionnement des écoles maternelles et élémentaires. 
DECRETO 149/2009, de 12 de mayo, por el que se regulan los centros que imparten el primer ciclo de la educación infantil. BOJA núm. 92, Sevilla, 15 de mayo 2009.

EUROSTAT (2012): Education and Training. European Commission, (http:// epp.eurostat.ec.europa.eu/portal/page/portal/education/introduction), consultado el 20 de mayo de 2012.

EUROSTAT (2012): Income and Living Conditions. European Commission, (http://epp.eurostat.ec.europa.eu/portal/page/portal/income social inclusion living conditions/introduction), consultado el 8 de mayo de 2012.

EURYDICE (2010a): Organisation of education system in France, 20092010 (Brussels, Eurydice).

EURYDICE (2010b): Organisation of education system in Germany, 20092010 (Brussels, Eurydice).

EURYDICE (2010c): Organisation of education system in United Kingdom, 2009-2010 (Brussels, Eurydice).

EURYDICE (2010d): Organización del sistema educativo español, 20092010 (Bruselas, Eurydice).

EURYDICE (2012): Key Data on Education in Europe (Brussels, Eurydice).

GARCÍA GARRIDO, J. L. (1989): Pasado y presente de la Educación Infantil, en VVAA, Pedagogía de la Escuela Infantil, pp. 33-64 (Madrid, Santillana).

LOCE: Ley Orgánica 10/2002, de 23 de diciembre (BOE de 24 de diciembre de 2002) de Calidad de la Educación.

LOE: Ley Orgánica 2/2006, de 3 de mayo (BOE de 4 de mayo de 2006) de Educación.

MECD (2012): Educación Infantil. Ministerio de Educación, Cultura y Deporte. Madrid. (http://www.educacion.gob.es/educacion/que-estudiary-donde/educacion-infantil.html), consultado el 5 de mayo de 2012.

MINISTÈRE DE L'ÉDUCATION NATIONALE (2012): L'école maternelle. Paris. (http://www.education.gouv.fr/cid166/l-ecole-maternelle- 
organisation- programme-et-fonctionnement.html), consultado el 3 de mayo de 2012.

OCDE (2001): Niños pequeños, grandes desafíos la educación y el cuidado de la infancia temprana (París, OCDE).

ORGANIZACIÓN DE LOS ESTADOS AMERICANOS (2009): Las transiciones en la primera infancia: una mirada internacional. Macro conceptual, situación, avances y desafíos (Washington, OEA/DEC).

PASSERIEUX, C. (Coord.) (2009): La Maternelle. Première École. Premiers Apprentissages (Lyon, Chronique Sociale).

REAL DECRETO 829/2003, de 27 de junio, por el que se establecen las enseñanzas comunes de la Educación Infantil. BOE núm. 156, 1 de julio de 2003.

REAL DECRETO 114/2004, de 23 de enero, por el que se establece el currículo de la Educación Infantil. BOE núm. 32, 6 febrero 2004.

REAL DECRETO 1630/2006, de 29 de diciembre, por el que se establecen las enseñanzas mínimas del segundo ciclo de Educación infantil. BOE núm. 4, 4 de enero 2007.

SANCHEZ A. (2007): Problemas de convivencia en la sociedad actual y repercusión en los centros escolares, en F. FULLEA y D. RIBAO (Coords.), Aprender a convivir desde el entorno escolar, pp. 37-56 (Madrid, El Corte Inglés).

SÁNCHEZ MULITERNO, J. (2009): El estado de la Educación Infantil en España. La opinión de los maestros y educadores infantiles. Estudios e Investigaciones, CEE Participación Educativa, 12, pp. 56-73.

SÁNCHEZ, A. (2008): Enfoque, estabilidad y continuidad de la escolarización en Educación Infantil (0-6 años). Evolución histórica en la Comunidad de Madrid, Revista de educación, 347, pp. 75-99.

SANCHIDRIÁN, C. y RUIZ BERRIO, J. (2010): Historia y perspectiva actual de la educación infantil (Barcelona, Grao).

SCHULGESETZ FÜR DAS LAND BERLIN (2010): Bildung für Berlin. 28 juni 2010. (Berlin). 
SCHULTE, B. (2005): El sistema educativo alemán, en J. PRATS y F. RAVENTÓS (Dirs.), Los sistemas educativos europeos ¿Crisis o transformación?, pp. 149-176 (Barcelona, Fundación La Caixa).

SYNDICAT DES ENSEIGNANTS DE FRANCE (2005): Code Soleil: Le système éducatif, carrières et status (Paris, Le Cherche Midi, Sudel).

TERCERO PUYOL, R. y ROVIRA BALLABRIGA, L. (2008): Proyecto de innovación e investigación educativa. La educación emocional como clave de un desarrollo saludable, (www.doredin.mec.es/documentos/ 00220091000023.pdf), consultado el 15 de mayo de 2012.

TORRES SÁNCHEZ, M. y GONZÁLEZ FARACO, J. C. (2008): La educación infantil en las culturas escolares europeas: un análisis político comparado, XXI Revista de Educación, 10, pp. 49-64.

\section{PROFESIOGRAFÍA:}

\section{Vicente Llorent Bedmar}

Profesor Titular de Educación Comparada en la Universidad de Sevilla. Director del Grupo de Investigación de Educación Comparada de Sevilla. Past-President de la Sociedad Española de Educación Comparada.

Datos de contacto: Facultad de Ciencias de la Educación. Universidad de Sevilla. Calle Pirotecnia, s/n. 41013, Sevilla (España). E-mail: $\underline{\text { llorent@us.es }}$

Fecha de recepción: 17 de julio de 2012

Fecha de revisión: 5 de septiembre de 2012 y 19 de septiembre de 2012

Fecha de aceptación: 10 de octubre de 2012 